\title{
A Note on Place Names
}

The Chinese words for jurisdictional units have been translated in the conventional manner: $f u$ is rendered as "prefecture" and xian as "county." The term xian is appended to place names only when the name would otherwise be monosyllabic or ambiguous. The term zhili zhou (literally, "independent district"), has been translated as "county" when it refers to a district encompassing a single county and "prefecture" when it refers to a district encompassing multiple counties (e.g., the four-county units centered on Linqing and Jining). The province north of Shandong, which was Zhili until 1927 and Hebei afterward, is identified by the usage of the period discussed in a given passage, or occasionally by Zhili/Hebei. The city of Liaocheng, once known as Dongchangfu, has been rendered as Liaocheng throughout, reserving the name Dongchangfu for the prefecture of which it was the capital. 
